\title{
Auger-Assisted Ultrafast Fluorescence Measurement of Semiconductor Single-Walled Carbon Nanotubes
}

Fengrui Hu, ${ }^{\dagger}$ Zengle Cao,${ }^{\dagger}$ Zheng Hua,${ }^{\dagger}$ Qinfeng Xu ${ }^{\dagger},{ }^{\dagger}$ Ming Zheng, ${ }^{\dagger}$ Chunfeng

Zhang, ${ }^{\dagger}$ Xiaoyong Wang, ${ }^{\dagger, *}$ and Min Xiao ${ }^{\dagger, \S, *}$

${ }^{\dagger}$ National Laboratory of Solid State Microstructures and School of Physics, Nanjing

University, Nanjing 210093, China

†Polymers Division, National Institute of Standards and Technology, Gaithersburg, MD 20899, USA

${ }^{\S}$ Department of Physics, University of Arkansas, Fayetteville, AR 72701, USA

${ }^{\#}$ Department of Physics and Optoelectronic Engineering, Ludong University, Yantai 264025, China 


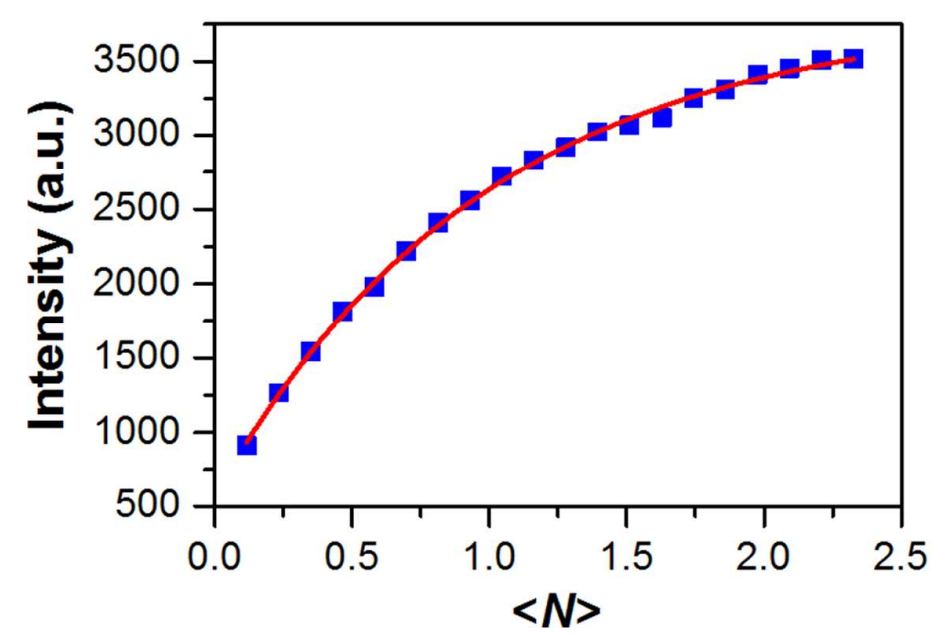

Figure S1. Spectrally-integrated PL intensity of $(6,5)$ SWCNTs plotted as a function of the exciton number $<N>$. In Figure 2c, the PL intensity $(I)$ of $(6,5)$ SWCNTs excited at $850 \mathrm{~nm}$ is plotted as a function of the single-pulse laser power $(P)$, which can be fitted by the function of $I \propto 1-\mathrm{e}^{-\alpha P}$. After getting the fitting constant $\alpha$, the number of excitons $\langle N\rangle$ generated per pulse in an individual SWCNT can be calculated from $<N>=\alpha P$ for a specific laser power $P$. 


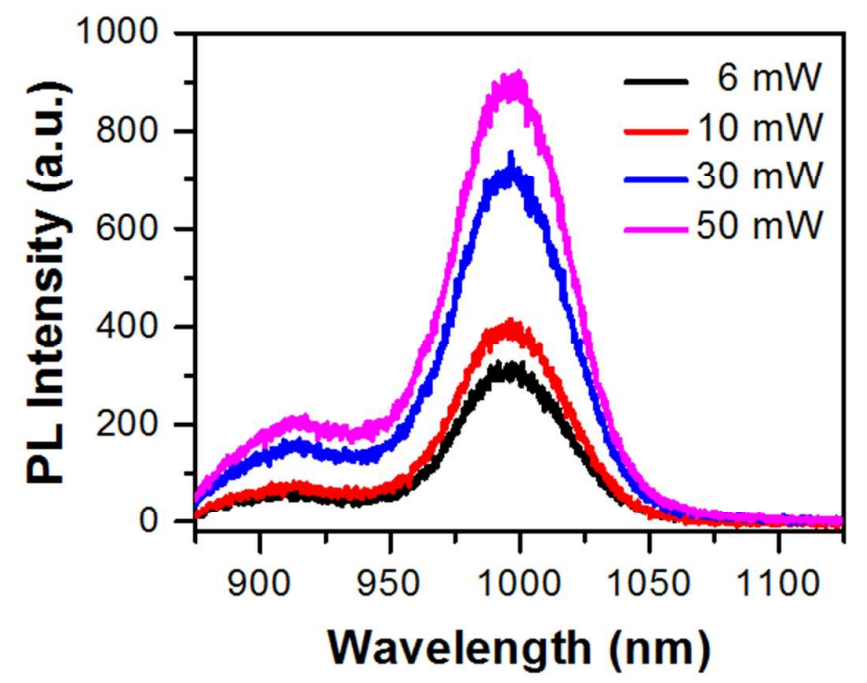

Figure S2. PL spectra of SWCNTs measured at four different laser powers. 


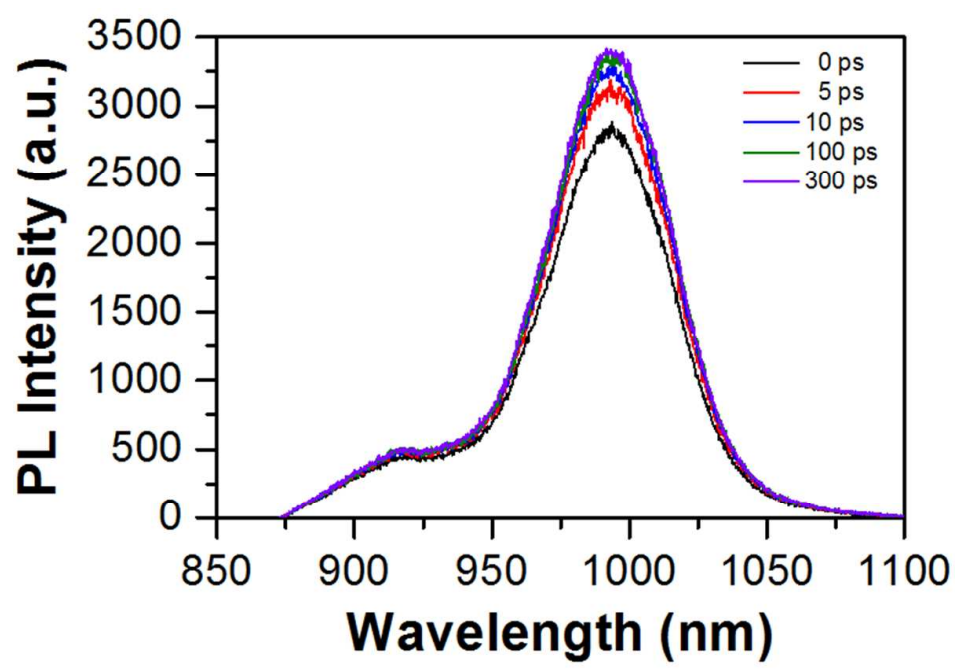

Figure S3. PL spectra of SWCNTs measured at several specific time delays between the two excitation laser pulses. The laser power at each beam was set at $30 \mathrm{~mW}$. 

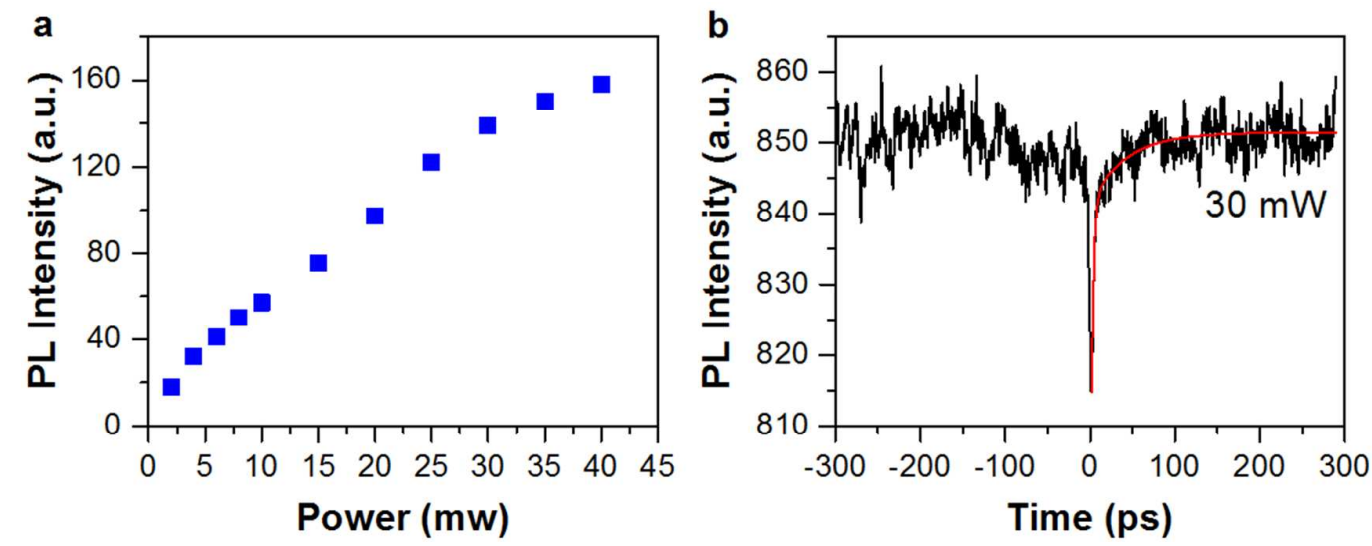

Figure S4. (a) Spectrally-integrated PL intensity of $(9,1)$ SWCNTs plotted as a function of the single-pulse laser power at the excitation wavelength of $\sim 850 \mathrm{~nm}$. (b) Spectrally-integrated PL intensity of $(9,1)$ SWCNTs plotted as a function of the time delay between the two excitation pulses. The laser wavelength was set at $\sim 850 \mathrm{~nm}$ and the laser power at each beam was $30 \mathrm{~mW}$. The solid line is a bi-exponential fitting with a fast and a slow lifetime component of $2.5 \mathrm{ps}$ and $41.3 \mathrm{ps}$, respectively. 

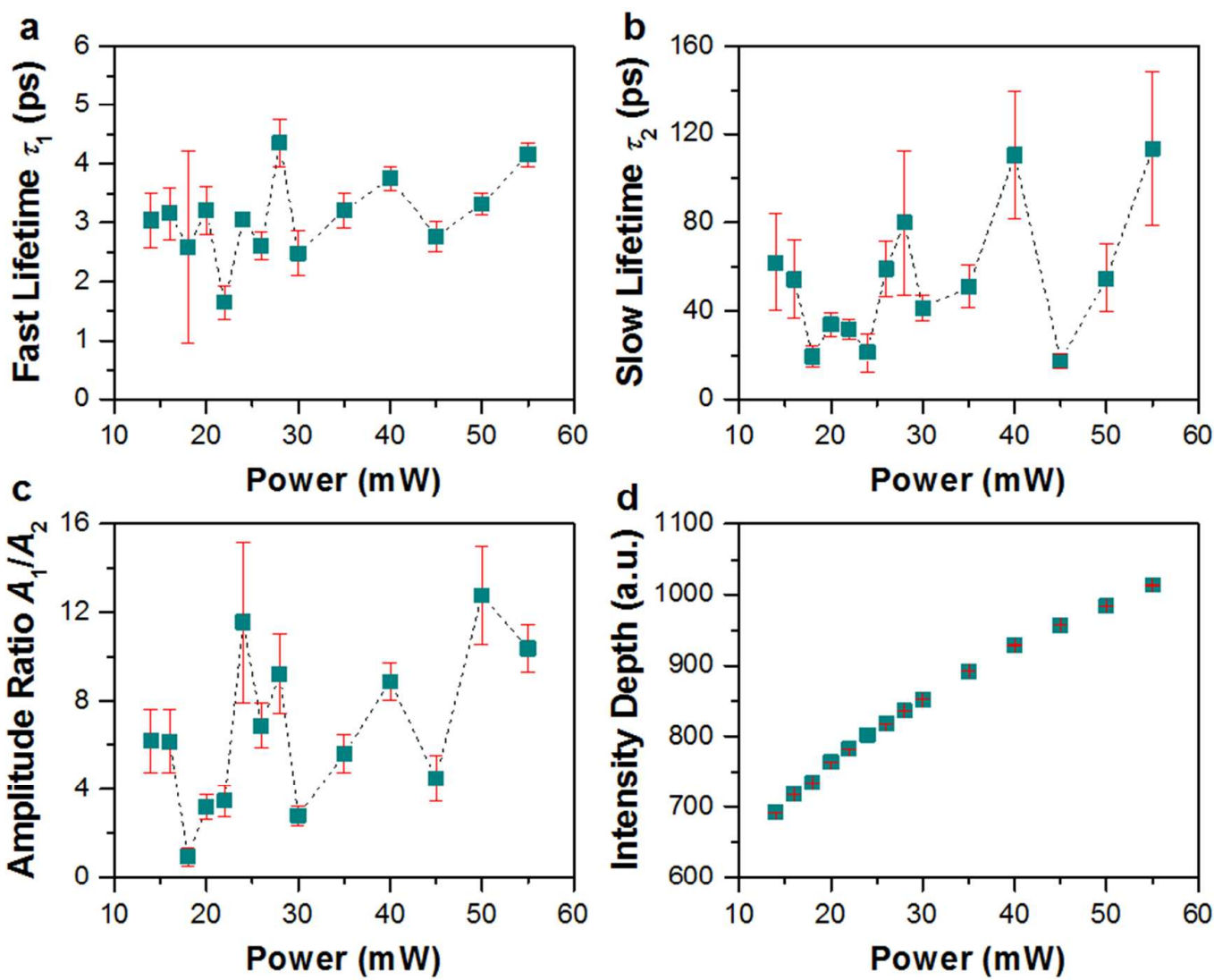

Figure S5. (a) The fast lifetime component $\tau_{1}$, (b) the slow lifetime component $\tau_{2}$, (c) the amplitude ratio $A_{1} / A_{2}$ between the fast and slow lifetime components, and (d) the PL intensity depth plotted as functions of the excitation laser power at each beam for the $(9,1)$ SWCNTs excited at $\sim 850 \mathrm{~nm}$. The dotted lines in (a), (b) and (c) are just guides to the eye. 


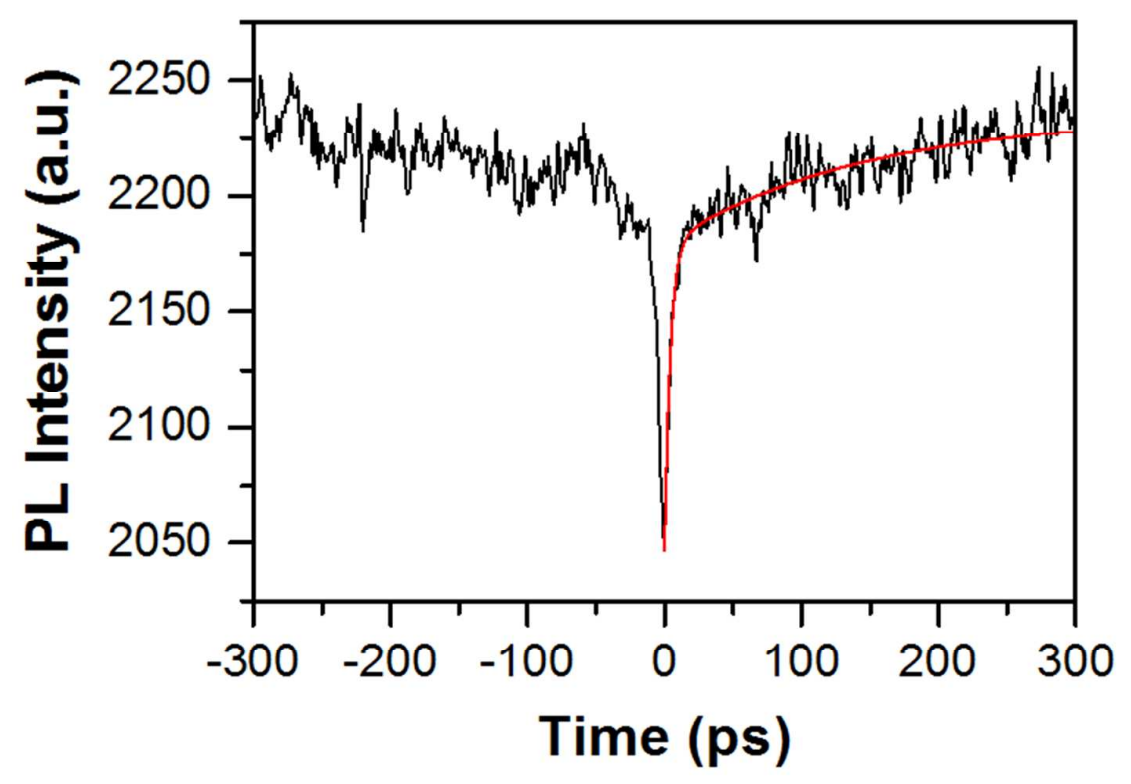

Figure S6. AUF curve measured for the $(6,5)$ SWCNTs in a sample highly enriched in the $(6,5)$ chirality. The solid line is a bi-exponential fitting with a fast and a slow lifetime component of 4.2 ps and 144 ps, respectively. 